Published in final edited form as:

Endocr Pract. 2015 April ; 21(4): 400-405. doi:10.4158/EP14512.RA.

\title{
Genetics of Primary Aldosteronism
}

Anand Vaidya, MD MMSc ${ }^{1}$, Amir H. Hamrahian, MD², and Richard J. Auchus, MD PhD ${ }^{3}$

${ }^{1}$ Center for Adrenal Disorders, Brigham and Women's Hospital, Harvard Medical School

${ }^{2}$ Department of Endocrinology, Cleveland Clinic Abu Dhabi

${ }^{3}$ Division of Endocrinology, University of Michigan Medical Center

\section{Abstract}

Objective-The AACE Adrenal Scientific Committee has developed a series of articles to update members on the genetics of adrenal diseases.

Methods-Case presentation, discussion of literature, table, and bullet points.

Results-Primary aldosteronism (PA) is the most common form of secondary hypertension. Early detection, surveillance, and treatment of PA may mitigate future cardiovascular risk. The genetics of PA are rapidly evolving and the consideration for genetic causes of PA are growing. Three inheritable forms of PA are now recognized: familial hyperaldosternism type I (glucocorticoid remediable aldosteronism), familial hyperaldosteronism type II, and familial hyperaldosteronism type III. The recent discovery of familial hyperaldosteornism type III spurred a flurry of international and collaborative research is identifying more genetic and molecular causes of PA that relate to mutations in membrane electrolyte transport channels of zona glomerulosa cells.

Conclusions-This article reviews the various genetic forms of PA including focus on the molecular mechanisms involved, diagnosis and treatment.

\section{Case Vignette}

A 25 year old man was evaluated for primary aldosteornism. When he was 14 years old, he was told he had a blood pressure of 148/92 $\mathrm{mmHg}$ during a routine sports physical examination; however, this finding was dismissed at the time. At age 22 years, he was noted to have a blood pressure of $160 / 90 \mathrm{mmHg}$ during a routine physical examination with his primary care physician. A thorough family history revealed that he had one 20 year-old brother who also had hypertension and one 18 year-old sister who was normotensive as far as he was aware. His father was diagnosed with hypertension at the age of 30 years and had severely uncontrolled blood pressure requiring more than 4 anti-hypertensive medications by the age of 45 years. One of his paternal uncles also had difficult to control hypertension diagnosed at an early age. His mother did not have hypertension. His paternal grandfather also had hypertension and may have died of a hemorrhagic stroke in his 5th decade of life,

Corresponding Author: Anand Vaidya, MD MMSc, Director, Center for Adrenal Disorders, Brigham and Women's Hospital, 221 Longwood Ave, RFB 287, Boston, MA, 02115, anandvaidya@bwh.harvard.edu. 
but this history could not be confirmed. The patient did not have further details on the medical history of his paternal uncles or aunts, or his paternal grandmother.

He was treated with hydrochlorothiazide but remained hypertensive. At age 25 years, his blood pressure was $186 / 102 \mathrm{mmHg}$ while on treatment with hydrochlorothiazide, and his primary care physician escalated therapy to include lisinopril and amlodipine. With this escalation, his blood pressure declined to $150 / 90 \mathrm{mmHg}$. An evaluation for secondary causes of hypertension revealed a serum potassium concentration of $4.1 \mathrm{mmol} / \mathrm{L}$, serum aldosterone concentration of $34 \mathrm{ng} / \mathrm{dL}$, and plasma renin activity of $0.3 \mathrm{ng} / \mathrm{mL} / \mathrm{h}$. The aldosterone-to-renin ratio (ARR) was calculated to be 113, which was interpreted to be very abnormal in the view of being on an ACE inhibitor. His estimated glomerular filtration rate was normal, and there was no microalbuminuria. Electrocardiogram was suggestive of left ventricular hypertrophy. A 24 hour urine collection after 3 days of oral salt loading revealed a 24 hour urinary sodium content of $208 \mathrm{mmol}$ with a concomitant urinary aldosterone excretion of $22 \mu \mathrm{g} / \mathrm{day}$. A diagnosis of primary aldosteronism was confirmed. Computed tomography of the abdomen revealed a 5-6 mm left adrenal gland nodule that was $<10$ Hounsfield units. The right adrenal gland was normal. Before proceeding with further localization with adrenal venous sampling, a diagnosis of glucocorticoid-remediable aldosteronism was considered.

- What testing should be recommended for this patient?

- How will further testing inform this patient's management?

\section{Discussion}

This patient has severe hypertension with testing confirming primary aldosteronism (PA) without a lateralizing source. PA is the most common form of secondary hypertension. It is conservatively estimated that $1-10 \%$ of the general hypertensive population, and up to 15$20 \%$ of the resistant hypertensive population, may have PA. Beyond the classical ramifications of excess aldosterone, including hypertension and hypokalemia, it is now recognized that subclinical and overt mineralocorticoid receptor activation contributes to significant cardiovascular and metabolic disease. Therefore, early detection and treatment of PA may mitigate future cardiovascular disease risk for this patient. Furthermore, given this patient's young age and family history of early onset hypertension and possible stroke, consideration for a heritable cause of PA should be considered, and a genetic diagnosis would implicate screening and treatment of potentially affected family members.

\section{Types of Primary Aldosteronism}

Traditionally, the categorization of PA has been largely based on the anatomic location of the excess aldosterone source: unilateral or bilateral. A unilateral aldosterone producing adenoma (APA) is often treated surgically, whereas bilateral adrenal hyperplasia (BAH) is generally treated medically with mineralocorticoid antagonist therapy. The concept that most cases of PA are sporadic is undergoing substantial scrutiny and evolution. For decades, the only inheritable forms of PA were familial hyperaldosteronism type I (FH-I), also known as glucocorticoid remediable aldosteronism (GRA), and familial hyperaldosteronism type II (FH-II) (Table). FH-I has a distinct and well understood genetic mechanism, whereas the 
genetic defect in FH-II is unknown; however, both are very rare. The estimated prevalence of FH-1 is $\sim 1 \%$ of PA cases and that of FH-2 is $\sim 6 \%$ of PA cases. More recently, the field of PA has undergone a renaissance period shepherded by advances in genetics that have lead to the discovery of mutated cell membrane channels on zona glomerulosa cells of the adrenal gland that contribute to PA (Table). While inherited causes of PA are still considered to be rare occurrences, our evolving understanding of the genetics of PA may implicate the more routine use of genetic technologies in the diagnosis, sub-typing, and familial screening of patients with PA.

- Familial Hyperaldosteronism type I (GRA): GRA is the result of a chimeric gene duplication resulting from unequal crossing over between the $11 \beta$-hydroxylase (CYP11B1) and aldosterone synthase genes (CYP11B2). Aldosterone is normally synthesized only in the zona glomerulosa due to the local expression of aldosterone synthase, which catalyzes the conversion of 11-deoxycorticosterone to corticosterone to aldosterone. This step is regulated mainly by angiotensin II and potassium; although corticotropin (ACTH) also stimulates aldosterone synthesis and therefore normal aldosterone secretion follows a diurnal pattern, like cortisol. In contrast, cortisol is normally synthesized only in the zona fasiculata where 11ßhydroxylase converts 11-deoxycortisol to cortisol. This step is regulated entirely by ACTH. In GRA, a chimeric gene is created from the recombination of the ACTHresponsive promoter region of the $11 \beta$-hydroxylase gene to coding sequences of the aldosterone synthase gene. In this context, a chimeric enzyme with aldosterone synthase activity is expressed entirely under the regulation of ACTH and ectopically in the zona fasciculata. As a result, aldosterone synthesis fluctuates with a diurnal rhythm in parallel with ACTH and does not respond to angiotensin II or potassium stimuli. Furthermore, because aldosterone synthase is expressed ectopically in the zona fasciculata, GRA is characterized by high quantities of cortisol metabolites hydroxylated at the C-18 position (18-oxocortisol, 18hydroxycortisol). The clinical presentation associated with these genetic and molecular abnormalities is generally: early age of hypertension onset, mild-tosevere hypertension, and early onset of cerebrovascular disease (such as hemorrhagic stroke). It is worth noting that a personal or family history of hypertension is not obligate in GRA, as some members of affected GRA kindreds demonstrate normal blood pressure or only mild hypertension. Furthermore, while some affected patients display hypokalemia with aldosterone excess, normokalemia is often seen with GRA. Therefore, normal blood pressure and potassium handling are not reliable criteria to dismiss the possibility of GRA.

- Familial Hyperaldosteronism type II: The genetic or molecular basis of FH-II has not been elucidated. By definition, FH-II refers to inheritable cases of PA (two or more affected family members with PA) that are not glucocorticoid remediable or explained by another genetic mechanism. There are no consistent demographic, biochemical, or imaging characteristics that herald FH-II. Notably, some cases of FH-II have been explained and re-defined as FH-III. Therefore, it remains to be seen whether cases of FH-II represent a heterogeneous collection of genetic forms of PA that are yet to be identified. 
- Familial Hyperaldosteronism type III: In 2008, a family with a unique form of early onset PA that exhibited high levels of C-18 oxygenated steroids, but was not glucocorticoid remediable, was reported. This description led investigators to identify a gain-of-function germline mutation in the KCNJ5 gene that encodes an inward-rectifying potassium channel on zona glomerulosa cells. Zona glomerulosa cells normally have a negative membrane resting potential that is largely mediated by potassium currents. Depolarization of the cell, either via angiotensin II stimulation or hyperkalemia-mediated inhibition of the potassium current, results in opening of voltage-gated calcium channels, increased intracellular calcium signaling, and stimulation of aldosterone synthase. Reported mutations in KCNJ5 result in a loss of potassium selectivity of the channel and permit the influx of sodium, thereby resulting in a more positive cell membrane potential (depolarization), which promotes calcium influx resulting in aldosterone synthesis. This new genetic mechanism of PA has been termed FH-III. Although germline mutations (inheritable mutations) of KCNJ5 are rare, multiple independent and international studies to date have described somatic mutations (tissue-specific mutations that are not transmitted to offspring) of KCNJ5 in up to $40 \%$ (and $>60 \%$ in Japanese cohorts) of APAs. Moreover, the discovery of a "channelopathy" as a genetic mechanism for heritable forms of PA focused investigators searching for new candidate genes in PA. Somatic and germline mutations in CACNAID, which encodes a voltage-gated calcium channel, have been reported to cause PA, as have somatic mutations in ATP1A1 and ATP2B3, a Na $/ \mathrm{K}^{+}$ATPase and a $\mathrm{Ca}^{2+}$-ATPase, respectively (Table).

\section{Biochemical, Imaging, and Genetic Testing for Primary Aldosteronism}

The biochemical diagnosis of all forms of PA requires the demonstration of autonomous and non-suppressible aldosterone secretion. This is typically performed with initial screening laboratories for serum aldosterone and plasma renin activity (PRA) to calculate an aldosterone-to-renin ratio (ARR). Patients may be evaluated while on their anti-hypertensive medications except for spironolactone and eplerenone which should be stopped for at least 4 weeks prior to the biochemical evaluation.

An ARR > 20 should raise the suspicion for PA, whereas an ARR>30, especially in the context of an aldosterone $>15 \mathrm{ng} / \mathrm{dL}$, is highly suspicious. The plasma aldosterone level should be considered when evaluating the ARR, since a PRA of $0.1 \mathrm{ng} / \mathrm{ml} / \mathrm{h}$, even in the presence of a serum aldosterone of only $3 \mathrm{ng} / \mathrm{dL}$, will result in an ARR of 30. A plasma aldosterone level $<9 \mathrm{ng} / \mathrm{dL}$ in the presence of normokalemia makes a diagnosis of primary aldosteronism highly unlikely. Interpretation of the ARR should always be made after confirming a relative suppression of PRA, which indicates autonomous aldosterone excess. Patients with borderline ARR values should have their level repeated in early morning after correction of hypokalemia and preferably while on no salt restriction.

Recent studies have raised awareness about the low sensitivity of the plasma aldosterone/ renin activity ratio, particularly in presence of drugs such as angiotensin-converting enzyme inhibitors which are known to decrease the ARR ratio. In addition some studies have 
suggested that older patients with FH-I may have entirely normal ARR values, thus reliance on ARR screening may be imperfect in certain family members of a kindred.

Confirmation of positive screens with either an oral salt suppression study or intravenous saline suppression study should be performed, although captopril challenges and fludrocortisone suppression tests can also be used. Once PA is confirmed, consideration for heritable causes of PA should be reconsidered. FH-I should be considered in patients with early-onset hypertension (age < 20 years) and/or a family history of PA with or without early-onset cerebrovascular accidents. FH-I kindreds have been reported to have considerable variation in clinical presentation, with some affected patients having normal blood pressure. Additionally, while hypokalemia can be seen in FH-I, many FH-I patients exhibit normokalemia. Patients with FH-I and FH-III typically have marked elevations in 24 hour urine collection for 18-oxocortisol and 18-hydroxycortisol, although these assays are not commonly available.

Confirmation of PA should be followed by localization studies, which includes crosssectional abdominal imaging such as computed tomography or magnetic resonance imaging. These images serve to phenotype the adrenal glands and to provide anatomic mapping for potential surgery. Regardless of imaging results, it has become increasingly evident that adrenal venous sampling in all cases of PA increases the likelihood of successfully distinguishing unilateral disease (APA) from bilateral disease (BAH). Patients with FH-I may have normal appearing adrenal glands on imaging or tumors in one or both adrenals, yet aldosterone production is always bilateral in FH-I. Patients with FH-II or FH-III may present with normal or nodular adrenals, and massively enlarged adrenal glands are sometimes found in FH-III.

If a familial form of hyperaldosteronism is suspected based on personal or family history, or urinary 18-oxygenated cortisol metabolites, one may proceed directly to genetic testing. A dexamethasone suppression test to evaluate for aldosterone suppression can be conducted prior to genetic testing in an attempt to evaluate whether a patient has glucocorticoidremediable (FH-I) or non-glucocorticoid remediable aldosteronism (FH-II or FH-III). The gold standard for diagnosing FH-I is sequencing of the chimeric CYP11B2/CYP11B1 gene. The genetic test can be obtained by contacting the International GRA registry and sending the blood sample to the Yale University School of Medicine according to the instructions at their website, or through other commercial labs that offer the test. The gold standard for diagnosing FH-III is sequencing to detect deleterious mutations in the KCNJ5 gene. There are no genetic tests for FH-II; rather, it is diagnosed by exclusion of FH-I and FH-III.

\section{Treatment of Primary Aldosteronism}

Treatment of FH-I is unique from all other forms of PA underscoring the importance of recognizing and diagnosing this entity. Exogenous glucocorticoids are used to lower ACTH levels and thereby lower aldosterone synthesis. The goal of therapy is to normalize blood pressure and/or hypokalemia. Excessive use of glucocorticoids, however can result in secondary adrenal insufficiency and/or exogenous Cushing's syndrome. Therefore, the lowest physiologic dose of glucocorticoids necessary to lower aldosterone and normalize blood pressure should be used. Monitoring plasma renin activity, serum aldosterone, and 18- 
oxygenated cortisol metabolites can provide objective measures of treatment effect. Longer acting glucocorticoids, such as dexamethasone and prednisone, are more effective at maintaining ACTH suppression and preventing frequent ACTH escape. One approach is to prescribe a single dose of dexamethasone or prednisone at bedtime to suppress the morning ACTH peak. Starting with low doses is recommended to avoid glucocorticoid excess $(0.125$ $\mathrm{mg}$ of dexamethasone qHS or $2.5 \mathrm{mg}$ of prednisone qHS). Higher doses can be considered in more severe cases, and mineralocorticoid receptor antagonists can also be added to the regimen to spare patients from adverse effects associated with higher doses of glucocorticoids. Treatment of FH-II does not significantly differ from non-heritable forms of PA. The use of surgery and mineralocorticoid receptor antagonists should be used based on clinical judgment after considering the severity of the aldosterone excess and the response to medical management. Almost all cases of FH-III will require bilateral adrenalectomy owing to the severity of hyperaldosteronism.

\section{Case Summary}

Given the patient's personal and family history suggestive of an inherited cause of PA, a diagnosis of FH-I was suspected. A 24 hour urine collection revealed markedly elevated levels of 18-oxocortisol and 18-hydroxycortisol, and the patient's serum aldosterone level was undetectable after taking an $8 \mathrm{mg}$ dose of dexamethasone the night before. A genetic test for the CYP11B2/CYP11B1 chimeric gene was sent, and results confirmed a diagnosis of FH-I. The patient was initiated on treatment $0.25 \mathrm{mg}$ of dexamethasone qHS and within a few weeks of therapy was able to titrate his blood pressure regimen down to just dexamethasone and low-dose lisinopril (the latter for presumed residual essential hypertension). He was counseled about the inheritance pattern of his autosomal-dominant disease and the risks of sustained untreated PA. His family members agreed to genetic testing, and his younger brother, father, and paternal uncle were all found to have FH-I as well.

\section{Selected References}

1. Brown JM, Underwood PC, Ferri C, et al. Aldosterone dysregulation with aging predicts renal vascular function and cardiovascular risk. Hypertension. 2014; 63:1205-1211. [PubMed: 24664291]

2. Funder JW, Carey RM, Fardella C, et al. Case detection, diagnosis, and treatment of patients with primary aldosteronism: an endocrine society clinical practice guideline. J Clin Endocrinol Metab. 2008; 93:3266-3281. [PubMed: 18552288]

3. Halperin F, Dluhy RG. Glucocorticoid-remediable aldosteronism. Endocrinol Metab Clin North Am. 2011; 40:333-341. viii. [PubMed: 21565670]

4. Vaidya, A.; Halperin, F.; Alexander, E.; Dluhy, RG. Hyperaldosteronism. 2014. wwwendotextorg

5. Zennaro MC, Rickard AJ, Boulkroun S. Genetics of mineralocorticoid excess: an update for clinicians. Eur J Endocrinol. 2013; 169:R15-R25. [PubMed: 23610123]

6. Lifton RP, Dluhy RG, Powers M, et al. A chimaeric 11 beta-hydroxylase/aldosterone synthase gene causes glucocorticoid-remediable aldosteronism and human hypertension. Nature. 1992; 355:262265. [PubMed: 1731223]

7. Geller DS, Zhang J, Wisgerhof MV, Shackleton C, Kashgarian M, Lifton RP. A novel form of human mendelian hypertension featuring nonglucocorticoid-remediable aldosteronism. J Clin Endocrinol Metab. 2008; 93:3117-3123. [PubMed: 18505761]

8. Choi M, Scholl UI, Yue P, et al. K+ channel mutations in adrenal aldosterone-producing adenomas and hereditary hypertension. Science. 2011; 331:768-772. [PubMed: 21311022] 
9. Scholl UI, Goh G, Stolting G, et al. Somatic and germline CACNA1D calcium channel mutations in aldosterone-producing adenomas and primary aldosteronism. Nature genetics. 2013; 45:1050-1054. [PubMed: 23913001]

10. Azizan EA, Poulsen H, Tuluc P, et al. Somatic mutations in ATP1A1 and CACNA1D underlie a common subtype of adrenal hypertension. Nature genetics. 2013; 45:1055-1060. [PubMed: 23913004]

11. Beuschlein F, Boulkroun S, Osswald A, et al. Somatic mutations in ATP1A1 and ATP2B3 lead to aldosterone-producing adenomas and secondary hypertension. Nature genetics. 2013; 45:440-444. 4e1-4e2. [PubMed: 23416519]

12. Carvajal CA, Campino C, Martinez-Aguayo A, et al. A new presentation of the chimeric CYP11B1/CYP11B2 gene with low prevalence of primary aldosteronism and atypical gene segregation pattern. Hypertension. 2012; 59:85-91. [PubMed: 22083159] 


\section{Key Take-Home Points}

- Primary aldosteronism is the most common form of secondary hypertension with an estimated prevalence of 1-10\% among the general hypertensive population, and up to $15-20 \%$ among the resistant hypertensive population

- Early detection and treatment of primary aldosteronism may improve blood pressure, reduce reliance on blood pressure medications, and mitigate future cardiovascular disease risk.

- Heritable causes of primary aldosteronism should be considered in patients diagnosed at a young age, those with a family history of primary aldosteronism, early-onset hypertension, or cerebrovascular accident.

- Familial hyperaldosteronism type I (glucocorticoid remediable aldosteronism) is a rare autosomal dominant disorder due to a chimeric gene duplication resulting from unequal crossing over between the 11 $\beta$-hydroxylase (CYP11B1) and aldosterone synthase genes (CYP11B2). Genetic testing confirms the diagnosis, and treatment with glucocorticoids can reduce ACTH-dependent aldosterone synthesis.

- Familial hyperaldosteronism type II is a heritable cause of primary aldosteronism defined by a family history of two or more affected family members with primary aldosteronism that are not glucocorticoid remediable or explained by another genetic mechanism. The molecular basis of this condition remains unknown.

- Familial hyperaldosteronism type III is a rare autosomal dominant form of primary aldosteronism caused by germline gain-of-fucntion mutations in the KCNJ5 gene; however, somatic mutations of KCNJ5 have been found in 40$60 \%$ of surgically resected aldosterone-producing adenomas. 


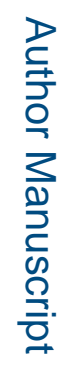

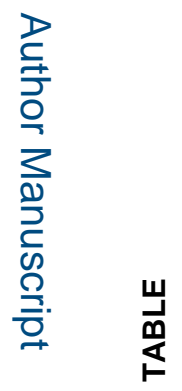

\begin{tabular}{|c|c|c|c|c|c|c|}
\hline & 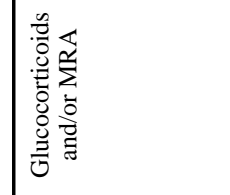 & 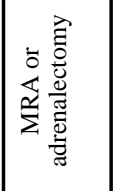 & 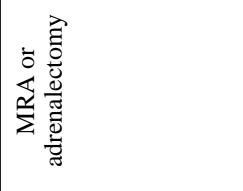 & 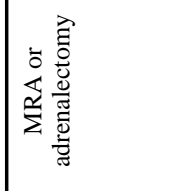 & 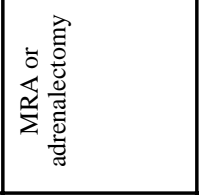 & 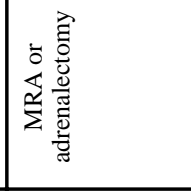 \\
\hline 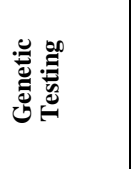 & 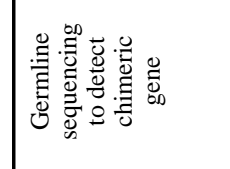 & 奇 & 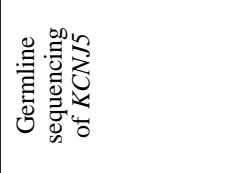 & 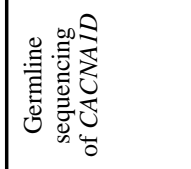 & & \\
\hline 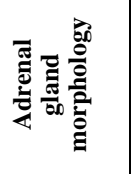 & 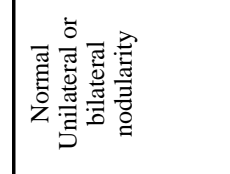 & 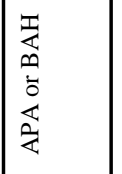 & 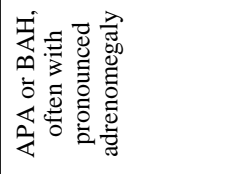 & 荇 & 蓆 & 荇 \\
\hline 噌 & 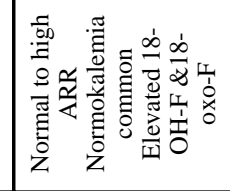 & 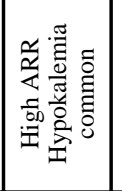 & 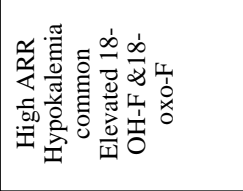 & 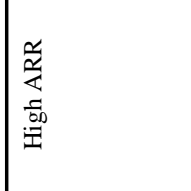 & 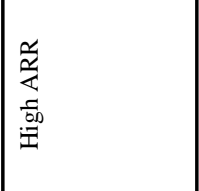 & 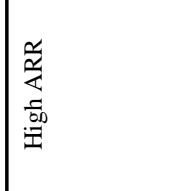 \\
\hline 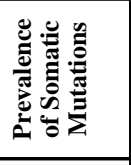 & , & & 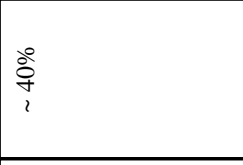 & $\frac{80}{2}$ & $\therefore$ & จั \\
\hline 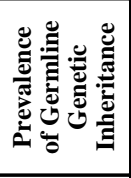 & 这 & & 这 & 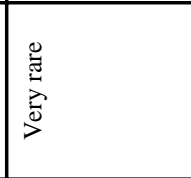 & 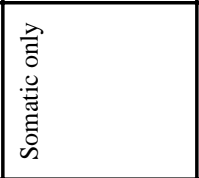 & 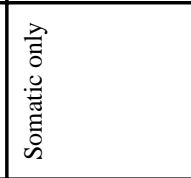 \\
\hline 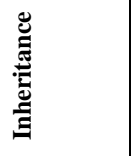 & \& & 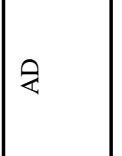 & \& & \& & & \\
\hline 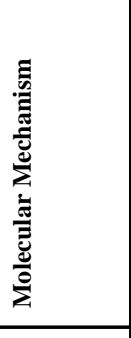 & 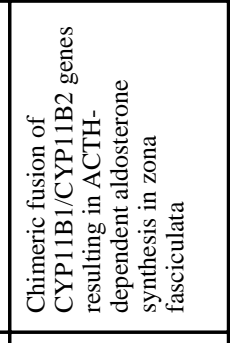 & 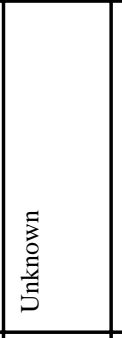 & 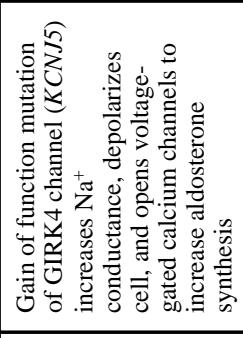 & 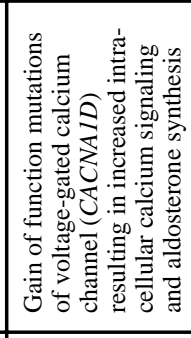 & 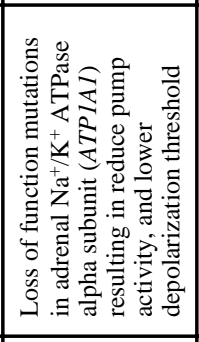 & 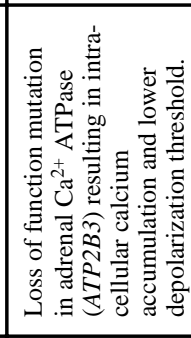 \\
\hline 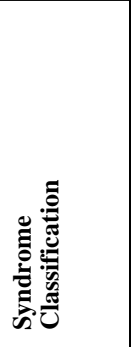 & 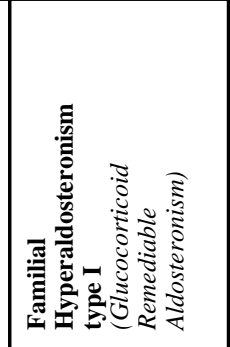 & 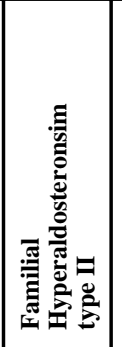 & 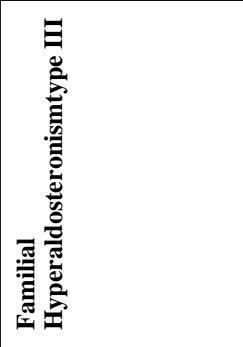 & & & \\
\hline
\end{tabular}

